\title{
Dissipations and Derivations
}

\author{
A. Kishimoto \\ Department of Physics, Kyoto Unıversity, Kyoto, Japan
}

\begin{abstract}
We show a usefulness of the notion of "dissipative operators" in the study of derivations of $C^{*}$-algebras and prove that the closure of a normal *-derivation of UHF algebra satisfying a special condition is a generator of a one-parameter group of *-automorphisms.
\end{abstract}

\section{$\S 1$. Introduction}

Recently various authors have studied unbounded derivations of $C^{*}$-algebras $[2-4,6,7,10,11,13]$. In particular Powers and Sakai [10] have studied unbounded derivations of UHF algebra.

The purpose of the present note is to show a usefulness of the notion of "dissipative operators" $[9,17]$ in the study of derivations of $C^{*}$-algebras.

Our first result is that an everywhere defined "dissipation" is bounded, which implies the well-known theorem concerning derivations $[5,12]$.

Our second result is about a normal *-derivation of UHF algebra satisfying a special condition discussed in $[1,10,14,15]$. For such a $*$-derivation, we prove that its closure is a generator of a one-parameter group of *-automorphisms. As its application we consider one-dimensional lattice system.

\section{§ 2. Bounded Derivation}

Let $\mathfrak{A}$ be a Banach space. For each $x \in \mathfrak{U}$ there is at least one non-zero element $f$ of the dual Banach space $\mathfrak{A}^{*}$ such that $\langle x, f\rangle=\|x\| \cdot\|f\|$ by the Hahn-Banach theorem. An $f_{x}$ denotes one of them throughout this note.

Definition 1. [9] A linear map $\gamma$ with domain $\mathscr{D}(\gamma)$ in a Banach space is called dissipative if there is an $f_{x}$ such that

$$
\operatorname{Re}\left\langle\gamma x, f_{x}\right\rangle \leqq 0
$$

for each $x \in \mathscr{D}(\gamma)$.

Definition 2. A linear map $\delta$ with domain $\mathscr{D}(\delta)$ in a Banach space is called derivative if there is an $f_{x}$ such that

$$
\operatorname{Re}\left\langle\delta x, f_{x}\right\rangle=0
$$

for each $x \in \mathscr{D}(\delta)$.

Let $\mathfrak{A}$ be a $C^{*}$-algebra. A linear map $\delta$ of $\mathfrak{U}$ is called a derivation if it satisfies

$$
\delta(x y)=\delta(x) y+x \delta(y)
$$


for $x, y \in \mathscr{D}(\delta)$, where $\mathscr{D}(\delta)$, the domain of $\delta$, is a *-subalgebra in $\mathfrak{A}$. A derivation $\delta$ is a *-derivation if $\delta(x)^{*}=\delta\left(x^{*}\right)$ for $x \in \mathscr{D}(\delta)$. In the following we will be concerned with only *-derivation and so omit *.

A linear map $\delta$ of $\mathfrak{A}$ is a derivation if $\delta$ and $-\delta$ are dissipations whose definition is:

Definition 3. [8] A linear map $\gamma$ of a $C^{*}$-algebra $\mathfrak{Q}$ is called a dissipation if it satisfies

$$
\begin{aligned}
& \gamma(x)^{*}=\gamma\left(x^{*}\right) \\
& \gamma\left(x^{*} x\right) \geqq \gamma\left(x^{*}\right) x+x^{*} \gamma(x)
\end{aligned}
$$

for each $x \in \mathscr{D}(\gamma)$, where $\mathscr{D}(\gamma)$, the domain of $\gamma$, is a *-subalgebra.

Remark 1. Call $\gamma$ an " $n$-dissipation" if $\gamma \otimes l ; \mathfrak{A} \otimes F_{n} \rightarrow \mathfrak{A} \otimes F_{n}$ is a dissipation where $F_{n}$ is an algebra of all $n \times n$ matrices and $l$ is an identity map. If $\gamma$ is a $2 n$ dissipation of a $C^{*}$-algebra with identity and $\mathscr{D}(\gamma) \ni 1$, then $\gamma^{\prime}$ defined by $\gamma^{\prime}(x)=$ $\gamma(x)-\frac{1}{2}\{\gamma(1) x+x \gamma(1)\}$ is an $n$-dissipation. Note $\gamma(1) \leqq 0$ and $\gamma^{\prime}(1)=0$. (See [8] for the arguments of bounded complete dissipations; a complete dissipation is defined to be an $n$-dissipation for all $n$.)

Lemma 1. Let $\gamma$ be a dissipation with domain $\mathscr{D}(\gamma)$. Suppose that for any positive $x \in \mathscr{D}(\gamma)$ there is an $f_{x}$ such that $\operatorname{Re}\left\langle\gamma x, f_{x}\right\rangle \leqq 0$. Then $\gamma$ is dissipative.

Proof. Note that $f_{x}$ is positive for a positive $x \in \mathfrak{A}$ [12]. If we define $f x^{*}$ and $x f$ in $\mathfrak{A}^{*}$ for $x \in \mathfrak{A}$ and $f \in \mathfrak{A}^{*}$ by $\left\langle a, f x^{*}\right\rangle=\left\langle x^{*} a, f\right\rangle$ and $\langle a, x f\rangle=\langle a x, f\rangle$ $(a \in \mathfrak{A})$, then $x f_{x^{*} x}=f_{x^{*}}$ and $f_{x^{*} x} x^{*}=f_{x^{*}}$. For any $x \in \mathscr{D}(\gamma)$, there is an $f=f_{x^{*} x}$ such that $\left\langle\gamma\left(x^{*} x\right), f\right\rangle \leqq 0$. Then we have

$$
\begin{aligned}
0 & \geqq\left\langle\gamma\left(x^{*} x\right), f\right\rangle \\
& \geqq\left\langle\gamma x^{*}, x f\right\rangle+\left\langle\gamma x, f x^{*}\right\rangle \\
& =2 \operatorname{Re}\left\langle\gamma x, f x^{*}\right\rangle .
\end{aligned}
$$

Lemma 2. (Lemmas 3.3 and 3.4 in [9]). A dissipative operator with dense domain in a Banach space is closable and its closure is also dissipative.

Sketch of the proof. Let $\gamma$ be the dissipative operator. Let $x_{n} \in \mathscr{D}(\gamma)$ with $x_{n} \rightarrow 0$ and $\gamma x_{n} \rightarrow y$. For any $a \in \mathscr{D}(\gamma)$ and $\lambda \in \mathbb{R}$, let $f_{n, \lambda}=f_{a+\lambda x_{n}}$ with $\left\|f_{n, \lambda}\right\|=1$ and $\operatorname{Re}\left\langle\gamma\left(a+\lambda x_{n}\right), f_{n, \lambda}\right\rangle \leqq 0$. We may suppose $f_{n, \lambda} \rightarrow f_{\lambda}(n \rightarrow \infty)$ and $f_{\lambda} \rightarrow f^{\prime}(\lambda \rightarrow \infty)$. Then we have $f^{\prime}=f_{a}$ and $\operatorname{Re}\left\langle y, f^{\prime}\right\rangle \leqq 0$. We may suppose $f^{\prime} \rightarrow f(a \rightarrow y)$. Then $f=f_{y}$ and $\|y\|=\operatorname{Re}\langle y, f\rangle \leqq 0$, i.e. $y=0$. The rest of the proof is easy.

In the rest of this section we will treat only everywhere defined operators.

Theorem 1. A dissipation $\gamma$ of a $C^{*}$-algebra $\mathfrak{A}(=\mathscr{D}(\gamma))$ is dissipative and bounded.

Proof. We suppose $\mathfrak{A} \ni 1$. If $\mathfrak{A} \not 1$, we can consider a dissipation $\gamma_{1}$ of $\mathfrak{A}_{1}=$ $\mathfrak{A}+\mathbb{C} \cdot 1$ defined by $\gamma_{1}(x+\lambda 1)=\gamma(x)(x \in \mathfrak{A}, \lambda \in \mathbb{C})$.

Let $x \in \mathfrak{A}$ be positive. Setting $h \equiv(\|x\| \cdot 1-x)^{1 / 2}$, we have for $f=f_{x}$,

$$
\begin{aligned}
\langle\gamma x, f\rangle & \leqq\langle\gamma(x-\|x\| \cdot 1), f\rangle \\
& =-\left\langle\gamma h^{2}, f\right\rangle \\
& \leqq-\langle(\gamma h) h, f\rangle-\langle h \gamma h, f\rangle \\
& =0
\end{aligned}
$$


where we have used the Schwartz inequality and the fact $\left\langle h^{2}, f\right\rangle=0$ and $f \geqq 0$. Hence $\gamma$ is dissipative by Lemma 1 and closed by Lemma 2. An everywhere defined closed operator is bounded by the closed graph theorem.

Corollary. A derivation of a $C^{*}$-algebra is derivative and bounded.

Proof. The proof is quite similar to the above. Or it follows from the above theorem by the following remark.

Remark 2. From the proof of Theorem 1 we can conclude that if $\gamma$ is a dissipation, for any $f_{x}, \operatorname{Re}\left\langle\gamma x, f_{x}\right\rangle \leqq 0$. It is immediate for $x \geqq 0$. For a general $x \in \mathfrak{A}$, any $f_{x}$ is equal to $f x^{*}$ where $f=f_{x^{*} x}=\|x\|^{-1}\left|f_{x}\right|$. (Let $x=u|x|$ the the polar decomposition of $x$ in the enveloping von Neumann algebra of $\mathfrak{A}$. Then $\left|f_{x}\right|=f_{x} u$, from which we can deduce $\left|f_{x}\right|=f_{|x|}=f_{x^{*} x}$.) The same situation prevails for derivations. (See Remark 2 in [9].)

Remark 3. [6] A dissipation $\gamma$ generates a uniformly continuous one-parameter semi-group of positive contractions $\Phi_{t}=e^{t \gamma}$. Lindblad showed the equivalence of (i) and (ii);

(i) $\Phi_{t}$ is uniformly continuous, $\Phi_{t}(1)=1$ and

$\Phi_{t}\left(x^{*}\right) \Phi_{t}(x) \leqq \Phi_{t}\left(x^{*} x\right)$.

(ii) $\gamma$ is a dissipation with $\gamma(1)=0$.

Finally we remark the following property of a derivation $\delta$. Let $x$ be selfadjoint and $C(x)$ be the commutative $C^{*}$-subalgebra generated by $x$ and 1 . Let $\varphi$ be a character of $C(x)$ and $\bar{\varphi}$ be any norm-preserving extension of $\varphi(\bar{\varphi}$ is a state). Then $\langle\delta x, \bar{\varphi}\rangle=0$ which is considered as generalization of derivativeness (see [5]).

This is easily seen; if a polynomial $P(x)$ of $x$ satisfies $\left\langle P^{\prime}(x), \varphi\right\rangle=P^{\prime}(\langle x, \varphi\rangle)=0$, then $\langle\delta P(x), \bar{\varphi}\rangle=0$. The set of such $P(x)$ is dense in $C(x)$ and so $\langle\delta x, \bar{\varphi}\rangle=0$ by the continuity of $\delta$.

\section{§ 3. Unbounded Derivations}

In the following the domain of a derivation or dissipation of a $C^{*}$-algebra is a dense *-subalgebra.

Theorem 2. Let $\gamma$ be a dissipation of a $C^{*}$-algebra $\mathfrak{A}$. If $\mathscr{D}(\gamma)$ is closed under the square root operation of positive elements, then $\gamma$ is dissipative and hence closable.

Proof $[4,10]$. The proof that $\gamma$ is dissipative is quite similar to that of Theorem 1. By Lemma 2 it is closable.

Let $\mathfrak{U}$ be a uniformly hyperfinite $C^{*}$-algebra (UHF algebra). A derivation $\delta$ in $\mathfrak{A}$ is said to be normal [10] if $\mathscr{D}(\delta)$ is the union of an increasing sequence of finite type I subfactors $\left\{\mathfrak{U}_{n} \mid n=1,2, \ldots\right\}$ in $\mathfrak{U}$.

Corollary. A normal derivation of a UHF algebra is derivative and hence closable. Its closure is also a derivative derivation.

Let $\tau$ be a unique tracial state on a UHF algebra $\mathfrak{A}$. A derivation $\delta$ in $\mathfrak{A}$ is said to be regular $[10]$ if $\langle\delta(a), \tau\rangle=0$ for $a \in \mathscr{D}(\delta)$.

Let $\delta$ be a normal derivation. Since $\langle a b, \tau \circ \delta\rangle=\langle b a, \tau \circ \delta\rangle$ for $a, b \in \mathscr{D}(\delta) \equiv \cup \mathfrak{A}_{n}$ and $\langle 1, \tau \circ \delta\rangle=0, \tau \circ \delta \mid \mathfrak{I}_{n}=0$ for any $n$. Hence $\delta$ is regular [10]. 
Theorem 3. If a derivation $\delta$ in a UHF algebra is regular, then $\delta$ is derivative.

Proof. Let $L^{2}(\mathfrak{A}, \tau)$ be a Hilbert space completion of a UHF algebra $\mathfrak{A}$ with inner product $\langle x, y\rangle_{\tau}=\left\langle y^{*} x, \tau\right\rangle$. Let $x$ be a positive element of $\mathscr{D}(\delta)$ and $L^{2}(C(x), \tau)$ be the closed subspace spanned by $C(x)$. Let $E_{x}$ be the orthogonal projection onto $L^{2}(C(x), \tau)$. If $\delta$ is regular,

$$
\begin{aligned}
0 & =\left\langle x^{n}, \tau \circ \delta\right\rangle \\
& =n\left\langle x^{n-1} \delta(x), \tau\right\rangle \\
& =n\left\langle\delta(x), x^{n-1}\right\rangle_{\tau} .
\end{aligned}
$$

Hence $E_{x} \delta(x)=0$. Let $\varphi$ be a character of $C(x)$ and $\hat{\varphi}$ be any norm-preserving extension of $\varphi$ into $L^{\infty}(C(x), \tau)^{*}$. Since $E_{x}: \mathfrak{U}\left(C L^{\infty}(\mathfrak{U}, \tau)\right) \rightarrow L^{\infty}(C(x), \tau)$ is a contraction, $\bar{\varphi}=\hat{\varphi} \circ E_{x}$ is an element of $\mathfrak{I}^{*}$. Let $\varphi$ be a character such that $\langle x, \varphi\rangle=$ $\|x\|\|\varphi\|=\|x\|$ and let $\bar{\varphi}=\hat{\varphi} \circ E_{x}$. Then $\bar{\varphi}=f_{x}$ and $\langle\delta x, \bar{\varphi}\rangle=0$. Now the proof is completed by Lemma 1 .

Let $\delta$ be a normal derivation in $\mathfrak{A}$. Let $\tilde{\delta}$ be the greatest linear extension of $\delta$ in all linear extensions $\gamma$ satisfying

$$
\begin{aligned}
& \gamma(a x b)=\delta(a) x b+a \gamma(x) b+a x \delta(b) \\
& \langle x, \tau \circ \gamma\rangle=0, \quad a, b \in \mathscr{D}(\delta), x \in \mathscr{D}(\gamma) .
\end{aligned}
$$

$\tilde{\delta}$ is called the greatest regular extension of a normal derivation $\delta$ [10].

Theorem 4. Let $\delta$ be a normal derivation. Suppose that $\tilde{\delta}$ is a derivation (or $\tilde{\delta}$ is derivative) and that there is an infinitesimal generator $\delta_{1}$ of a strongly continuous group of *-automorphisms such that $\delta_{1} \supseteqq \delta$. Then $\delta_{1}=\tilde{\delta}$.

Proof. Since $\delta_{1}$ is regular [10], $\delta_{1} \subseteq \tilde{\delta}$. As $(1 \pm \tilde{\delta}) \mathscr{D}(\tilde{\delta}) \supseteqq\left(1 \pm \delta_{1}\right) \mathscr{D}\left(\delta_{1}\right)=\mathfrak{A}$ and $\tilde{\delta}$ is derivative by Theorem $3, \tilde{\delta}$ is an infinitesimal generator by the following theorem and remark. Hence $\delta_{1}=\tilde{\delta}$.

Theorem 5. Let $\delta$ be a derivation of a $C^{*}$-algebra $\mathfrak{A}$. If $\delta$ is derivative and closed and $(1 \pm \delta) \mathscr{D}(\delta)$ is dense in $\mathfrak{A}$, then $\delta$ is an infinitesimal generator of a strongly continuous group of *-automorphisms.

Proof. If $f_{x}$ satisfies $\operatorname{Re}\left\langle\delta x, f_{x}\right\rangle=0$ and $\left\|f_{x}\right\|=1$,

$$
\begin{aligned}
\|(\delta+\lambda) x\| & \geqq \pm \operatorname{Re}\left\langle(\delta+\lambda) x, f_{x}\right\rangle \\
& = \pm \operatorname{Re} \lambda\|x\|
\end{aligned}
$$

i.e. $\|(\delta+\lambda) x\| \geqq|\operatorname{Re} \lambda| \cdot\|x\|$.

The rest of the proof is standard [2-4].

Remark 4. The assumption that $\delta$ is a derivation in Theorem 5 can be replaced as follows: Let $\delta$ be a linear operator with dense domain $\mathscr{D}(\delta)$ such that $\mathscr{D}(\delta) \ni 1$ and $\delta(1)=0$. It is shown as follows: By a result in the Hill-Yosida semi-group theory [17] $\delta$ generates a strongly continuous group of contractions $\varrho_{t}$ on $\mathfrak{A}$. Since $\varrho_{t}(1)=1$ (by the assumption $\delta(1)=0$ ) and $\left\|\varrho_{t}\right\|=1$ they are positive contractions. As they form a group, they are order-isomorphisms. Thus $\varrho_{t}$ is a strongly continuous one-parameter group of Jordan automorphisms [cf. 16]. Then it is known [18, Theorem 3.4] that $\varrho_{t}$ is a group of ${ }^{*}$-automorphisms. 
Remark 5. $\tilde{\delta}$ is in general not a derivation (see Problem 1 of [10]). For if $\delta$ is a normal derivation which has more than two different extensions to infinitesimal generators, then $\tilde{\delta}$ is not a derivation, as easily shown by using Theorem 4 . (We can construct such $\delta$. See Remark 3 of [10].)

Let $P_{n}$ be the canonical conditional expectation of $\mathfrak{A}$ onto $\mathfrak{A}_{n}$. Let $h_{n}$ be a self-adjoint element of $\mathfrak{U}$ such that $\delta(a)=\left[i h_{n}, a\right] \equiv \delta_{i h_{n}}(a)$ for all $a \in \mathfrak{U}_{n}$. Then $P_{n} \tilde{\delta}(x)=P_{n} \delta_{i h_{n}}(x)$ for $x \in \mathscr{D}(\tilde{\delta})[10]$. For if $a \in \mathfrak{A}_{n}$,

$$
\begin{aligned}
\left\langle a P_{n} \tilde{\delta}(x), \tau\right\rangle & =\langle a \tilde{\delta}(x), \tau\rangle \\
& =\langle a x, \tau \circ \tilde{\delta}\rangle-\langle(\delta a) x, \tau\rangle \\
& =-\left\langle\left(\delta_{i h_{n}} a\right) x, \tau\right\rangle \\
& =\left\langle a \delta_{i h_{n}} x, \tau\right\rangle \\
& =\left\langle a P_{n} \delta_{i h_{n}} x, \tau\right\rangle .
\end{aligned}
$$

In $[10] W \subset \mathscr{D}(\tilde{\delta})$ is defined by

$W \equiv\left\{x \in \mathscr{D}(\tilde{\delta}) ; \lim P_{n} \tilde{\delta}\left(1-P_{n}\right) x=0\right\}$.

If we set $P_{n}\left(h_{n}\right)=k_{n}$,

$$
W=\left\{x \in \mathscr{D}(\tilde{\delta}) ; \lim \delta_{i k_{n}} P_{n} x=\tilde{\delta}(x)\right\} .
$$

In [6] an operator ex-lim $\delta_{i k_{n}}\left(\right.$ the extended limit of the $\delta_{i k_{n}} \mid \mathfrak{A}_{n}$ ) is defined, whose graph is the set of $(x, y) \in \mathfrak{A} \times \mathfrak{A}$ such that there is a sequence $x_{n} \in \mathfrak{A}_{n}$, with $\left\|x_{n}-x\right\| \rightarrow 0$ and $\left\|\delta_{i k_{n}}\left(x_{n}\right)-y\right\| \rightarrow 0$.

In [7] an operator $\delta$ (the graph limit of the $\delta_{i k_{n}}$ ) is defined, whose graph is the set of $(x, y) \in \mathfrak{A} \times \mathfrak{A}$ such that there is a sequence $x_{n} \in \mathfrak{A}$, with $\left\|x_{n}-x\right\| \rightarrow 0$ and $\left\|\delta_{i k_{n}}\left(x_{n}\right)-y\right\| \rightarrow 0$.

Then

$\delta \subset \tilde{\delta} \mid W \subset \operatorname{ex}-\lim \delta_{i k_{n}} \subset \hat{\delta} \subset \tilde{\delta}$.

Theorem 6. $\hat{\delta}$ is derivative.

Proof. Let $x \in \mathscr{D}(\hat{\delta})$ and $\left\{x_{n}\right\}$ be a sequence such that $x_{n} \rightarrow x$ and $\delta_{i k_{n}}\left(x_{n}\right) \rightarrow \hat{\delta}(x)$. Let $f_{n}=f_{x_{n}}$ be of norm 1. We may suppose $f_{n} \rightarrow f$. Then $f=f_{x}$ and

$$
\begin{aligned}
\operatorname{Re}\langle\hat{\delta} x, f\rangle & =\lim \operatorname{Re}\left\langle\delta_{i k_{n}} x_{n}, f_{n}\right\rangle \\
& =0
\end{aligned}
$$

where we have used Remark 2.

Remark 6. $[6,7] \hat{\delta}$ and ex-lim $\delta_{i k_{n}}$ are closed derivations.

Lemma 3. If $\left\{\left\|h_{n}-k_{n}\right\|\right\}$ is uniformly bounded, $\tilde{\delta}$ is derivative.

Proof. Let $x \in \mathscr{D}(\tilde{\delta})$ and $f_{n}=f_{P_{n} x}$ with $\left\|f_{n}\right\|=1$. We may suppose $f_{n} \rightarrow f$. Then $f=f_{x}$ and

$$
\begin{aligned}
\operatorname{Re}\langle\tilde{\delta} x, f\rangle & =\lim \operatorname{Re}\left\langle P_{n} \tilde{\delta} x, f_{n}\right\rangle \\
& =\lim \operatorname{Re}\left\langle P_{n} \tilde{\delta}\left(1-P_{n}\right) x, f_{n}\right\rangle \\
& =\lim \operatorname{Re}\left\langle P_{n} \delta_{i h_{n}-i k_{n}}\left(1-P_{n}\right) x, f_{n}\right\rangle
\end{aligned}
$$


where we have used $\operatorname{Re}\left\langle P_{n} \tilde{\delta} P_{n} x, f_{n}\right\rangle=0, P_{n} \delta_{i k_{n}}\left(1-P_{n}\right)=0$ and $\delta_{i h_{n}-i k_{n}}=\delta_{i h_{n}}-\delta_{i k_{n}}$. The last term is dominated by

$$
2\left\|h_{n}-k_{n}\right\| \cdot\left\|\left(1-P_{n}\right) x\right\|
$$

which tends to zero as $n \rightarrow \infty$.

Theorem 7. Let $\delta$ be a normal derivation. If $\left\{\left\|h_{n}-k_{n}\right\|\right\}$ is uniformly bounded, $\bar{\delta}$, the closure of $\delta$, is an infinitesimal generator of a strongly continuous group of

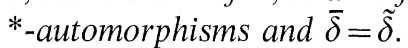

Proof. Suppose that $(1+\delta) \mathscr{D}(\delta)$ is not dense in $\mathfrak{A}$. Then there is an element $f$ in $\mathfrak{U}^{*}$ such that $\|f\|=1$ and $\langle x+\delta x, f\rangle=0$ for all $x \in \mathscr{D}(\delta)$. There are $x_{n} \in \mathfrak{U}_{n} \subset$ $\mathscr{D}(\delta) \equiv \cup \mathfrak{U}_{n}$ such that $\left\langle x_{n}, f\right\rangle=\left\|x_{n}\right\|\left\|f\left|\mathfrak{U}_{n}\|=\| f\right| \mathfrak{U}_{n}\right\|$. Then

$$
\begin{aligned}
0 & =\lim \operatorname{Re}\left\{\left\langle x_{n}, f\right\rangle+\left\langle\delta x_{n}, f\right\rangle\right\} \\
& =\lim \operatorname{Re}\left\{\left\|f \mid \mathfrak{A}_{n}\right\|+\left\langle\delta_{i h_{n}} x_{n}, f\right\rangle\right\} \\
& =\|f\|+\lim \operatorname{Re}\left\langle\delta_{i h_{n}-i k_{n}} x_{n}, f\right\rangle \\
& \geqq 1-\varlimsup \lim 2 \cdot\left\|h_{n}-k_{n}\right\|
\end{aligned}
$$

where we have used $\operatorname{Re}\left\langle\delta_{i k_{n}} x_{n}, f\right\rangle=0$. Suppose $\left\|h_{n}-k_{n}\right\|<1 / 2-\varepsilon(\varepsilon>0)$. Then it is a contradiction and hence $(1+\delta) \mathscr{D}(\delta)$ is dense in $\mathfrak{A}$. Quite similarly we can conclude that $(1-\delta) \mathscr{D}(\delta)$ is dense in $\mathfrak{A}$. Since $\bar{\delta}$ is derivative by Corollary of Theorem 3 , $\bar{\delta}$ is an infinitesimal generator by Theorem 5. If $\left\|h_{n}-k_{n}\right\|<C$ for any $n$, we may consider $\delta / 3 C$ instead of $\delta . \bar{\delta}=\tilde{\delta}$ follows from Theorem 4 and Lemma 3.

Remark 7. Under the assumption of Theorem 7 the one-parameter group $\varrho_{t}$ generated by $\bar{\delta}$ satisfies

$$
\varrho_{t}(x)=\lim e^{t \delta i k_{n}}(x), \quad x \in \mathfrak{U}
$$

where the convergence is uniform in $t$ on every compact subset of $(-\infty, \infty)$. This follows from Theorem 7 combined with Theorems 6 and 8 in [10] (cf. the proof of Theorem 8 below).

As an application of Theorem 7, we consider one-dimensional lattice system. Let $\left\{\mathfrak{U}_{j}: j \in Z\right\}$ be a family of type I finite factors and let $\mathfrak{U}=\bigotimes_{j \in Z} \mathfrak{U}_{j}$ be the infinite tensor product of them. Let $\Phi$ be a map from the family $P_{f}(Z)$ of finite subsets of $Z$ into $\mathfrak{A}$ such that $\Phi(\emptyset)=0$ and $\Phi(\Lambda)$ is a self-adjoint element of $\mathfrak{U}(\Lambda)=\bigotimes \underset{j \in \Lambda}{\bigotimes} \mathfrak{U}_{j}$. Put

$$
\|\Phi\|_{\alpha}=\sup _{j} \sum_{\Lambda \ni j} e^{\alpha N(\Lambda)}\|\Phi(\Lambda)\|
$$

where $N(\Lambda)$ denotes the number of points in $\Lambda$ and $\alpha \geqq 0$.

It is known (cf. [1]) that if $\|\Phi\|_{\alpha}<\infty$ for $\alpha>0$, there exists a one-parameter group of *-automorphisms such that

$$
\begin{aligned}
\varrho_{t}(Q) & =\lim _{\Lambda} e^{i t U(\Lambda)} Q e^{-i t U(\Lambda)}=\lim e^{t \delta i U(\Lambda)} Q, \quad Q \in \mathfrak{A} \\
U(\Lambda) & =\sum_{J \subset \Lambda} \Phi(J) .
\end{aligned}
$$


Now we give another sufficient condition for the existence of the above automorphism group:

Theorem 8. Suppose that (i) $\|\Phi\|_{0}<\infty$ and (ii) there is an increasing sequence $\left\{\Lambda_{n}\right\} \subset P_{f}(Z)$ such that $\cup \Lambda_{n}=Z$ and the following element $W\left(\Lambda_{n}\right)$ of $\mathfrak{U}$ is bounded in norm uniformly in $n$ :

$$
W\left(\Lambda_{n}\right)=\sum_{J}\left\{\Phi(J) ; J \in P_{f}(Z), J \cap \Lambda \neq \emptyset, J \cap \Lambda^{c} \neq \emptyset\right\}
$$

where $\Lambda^{c}$ denotes the complement of $\Lambda$ in $Z$. Then there exists a strongly continuous one-parameter group of *-automorphisms such that

$$
\varrho_{t}(Q)=\lim _{n} e^{t \delta_{n}}(Q)
$$

where $\delta_{n}=\delta_{i U\left(\Lambda_{n}\right)}$ and the convergence is uniformly in $t$ on every compact interval of $t$.

Proof. By (i), $W\left(\Lambda_{n}\right)$ is well-defined. Let $\mathfrak{U}_{n}=\mathfrak{A}\left(\Lambda_{n}\right)$ and let $h_{n}=U\left(\Lambda_{n}\right)+W\left(\Lambda_{n}\right)$. Let $\delta$ be the normal derivation such that

$$
\delta \mid \mathfrak{U}_{n}=\delta_{i h_{n}}, \quad \mathscr{D}(\delta)=\cup \mathfrak{U}_{n} .
$$

Then [1]

$$
\begin{aligned}
\left\|h_{n}-k_{n}\right\| & \leqq\left\|h_{n}-U\left(\Lambda_{n}\right)\right\|+\left\|U\left(\Lambda_{n}\right)-k_{n}\right\| \\
& \leqq 2\left\|W\left(\Lambda_{n}\right)\right\|
\end{aligned}
$$

where $k_{n}=P_{n}\left(h_{n}\right)$. Hence $\bar{\delta}$ is an infinitesimal generator by Theorem 7 . Now the proof of the convergence in $(*)$ follows as in [10]: It is shown by (i) that $\lim \delta_{n}=\delta$ on $\mathscr{D}(\delta)$. Then for $x \in \mathscr{D}(\delta)$

$$
\begin{aligned}
& \left\|\left\{\left(1 \pm \delta_{n}\right)^{-1}-(1 \pm \bar{\delta})^{-1}\right\}(1 \pm \bar{\delta}) x\right\| \\
& =\left\|\left(1 \pm \delta_{n}\right)^{-1}\left\{(1 \pm \bar{\delta}) x-\left(1 \pm \delta_{n}\right) x\right\}\right\| \\
& \leqq \\
& \quad \leqq(1 \pm \bar{\delta}) x-\left(1 \pm \delta_{n}\right) x \| \\
& \leqq \\
& \quad \rightarrow 0 \text { x } x-\delta_{n} x \| \\
& \quad \rightarrow 0 \text { as } n \rightarrow \infty .
\end{aligned}
$$

where we have used $\left\|\left(1 \pm \delta_{n}\right)^{-1}\right\| \leqq 1$.

Hence $\lim \left(1 \pm \delta_{n}\right)^{-1}=(1 \pm \bar{\delta})^{-1}$ since $(1 \pm \delta) \mathscr{D}(\delta)$ is dense in $\mathfrak{A}$. By the TrotterKato theorem [cf. 17] we get $(*)$.

Finally we remark that the assumption (i) can be weakened by (i') $\sum_{\Lambda \ni j}\|\Phi(\Lambda)\|<\infty$ for any $j \in Z$.

Acknowledgements. The author is deeply indebted to Professor H. Araki for his encouragement and useful suggestions. He would like to thank Professor H. Hasegawa for his encouragement. 


\section{References}

1. Araki, H.: Commun. math. Phys. 44, 1-7 (1975)

2. Bratteli, O.: Self-adjointness of unbounded derivations of $C^{*}$-algebras

3. Bratteli, O., Robinson, D. W.: Commun. math. Phys. 42, 253-268 (1975)

4. Bratteli, O., Robinson, D. W.: Unbounded derivations of $C^{*}$-algebras II

5. Diximier, J.: Les algebres d'operateurs dans l'espace hilbertien. Paris: Gauthier-Villars 1957

6. Herman, R.H.: Unbounded derivations

7. Herman, R.H.: Unbounded derivations II

8. Lindblad, G.: On the generator of quantum dynamical semi-groups

9. Lumer, G., Phillips, R. S. : Pacific J. Math. 11, 679-698 (1961)

10. Powers, R.T., Sakai, S.: J. Funct. Anal. 19, 81-95 (1975)

11. Powers, R.T., Sakai, S.: Commun. math. Phys. 39, 273-288 (1975)

12. Sakai, S.: $C^{*}$-algebras and $W^{*}$-algebras. Berlin-Heidelberg-New York: Springer 1970

13. Sakai, S.: On one parameter groups of *-automorphisms on operator algebras and the corresponding unbounded derivations. (to appear in Amer. J. Math.)

14. Sakai, S.: Commutative normal *-derivations II

15. Sakai, S.: Commutative normal *-derivations III

16. Stormer,E.: In: Lecture Notes in Physics 29. Berlin-Heidelberg-New York 1974

17. Yosida, K.: Functional Analysis. Berlin-Heidelberg-New York: Springer 1965

18. Kadison, R.V.: Topology 3, Suppl. 2, 177-198 (1965)

Communicated by H. Araki

(Received August 22, 1975) 